\title{
Exoesqueleto para rehabilitación de miembro inferior con dos grados de libertad orientado a pacientes con accidentes cerebrovasculares
}

\section{Exoskeleton for rehabilitation of the lower limb with two degrees of freedom for stroke patients with cerebrovascular accidents}

\author{
DOI: : http://doi.org/10.17981/ingecuc.15.2.2019.04
}

Artículo de Investigación Científica. Fecha de Recepción: 24/09/2018. Fecha de Aceptación:04/05/2019.

\author{
Diego Alexander Tibaduiza-Burgos \\ Universidad Nacional de Colombia, Bogotá. (Colombia) \\ dtibaduizab@unal.edu.co \\ Pedro Antonio Aya-Parra \\ Universidad del Rosario. Bogotá (Colombia) \\ pedro.aya@urosario.edu.co \\ Maribel Anaya-Vejar \\ Universidad Santo Tomás. Bogotá (Colombia) \\ maribelanaya@usantotoma.edu.co
}

Para citar este artículo:

Diego Alexander Tibaduiza-Burgos; Maribel Anaya-Vejar; Pedro Antonio Aya-Parra "Exoesqueleto para rehabilitación de miembro inferior con dos grados de libertad orientado a pacientes con accidentes cerebrovasculares" INGE CUC, vol. 15, no. 2, pp. 36-46, 2019. DOI: http://doi.org/10.17981/ingecuc.15.2.2019.04

\section{Resumen}

Introducción- Un exoesqueleto puede ser entendido como una estructura mecatrónica con la capacidad de ser acoplada a una extremidad de manera externa y la cual permite el desarrollo de los movimientos en las diferentes articulaciones de esta misma. Estos movimientos son desarrollados con el apoyo de actuadores que definen los grados de libertad de este tipo de dispositivos.

Objetivo- Dada su versatilidad, estas estructuras pueden llegar a ser una herramienta útil en la asistencia de trabajos de rehabilitación de miembros superior e inferior humanos, y en algunos casos, como este trabajo por ejemplo, ayudar a pacientes con parálisis en sus miembros para el desarrollo de actividades tan complejas como la marcha humana.

Metodología- Aunque hay algunos adelantos importantes en esta área, esta sigue en fase de investigación y muchos de los desarrollos aún no están disponibles o no son asequibles para su uso masivo en países como Colombia y en general América Latina. Desde este punto de vista y como contribución al desarrollo de esta área en el país, este trabajo presenta el desarrollo de un exoesqueleto activo, el cual fue diseñado para ayudar en la rehabilitación de pacientes que han tenido algún tipo de secuela como consecuencia de un Accidente Cerebro Vascular (ACV), también conocido como Ictus.

Resultados- Se incluye dentro del artículo, información sobre el modelado, el diseño, control y la construcción del dispositivo exoesquelético, así como el desarrollo de unas pruebas preliminares orientadas a mostrar su uso en el desarrollo de pruebas de repetición en el plano sagital.

Conclusiones- El resultado de estos análisis muestra el potencial del sistema desarrollado para su aplicación en este tipo de terapias de rehabilitación.

Palabras clave- Exoesqueleto; accidente cerebro vascular; rehabilitación; miembro inferior; control; prototipo.

\section{Abstract}

Introduction- An exoskeleton can be understood as a mechatronic structure with the capacity to be coupled to an external extremity and which allows the development of the movements in the different articulations of the same one. These movements are developed with the support of actuators that define the degrees of freedom of this type of device.

Objective- Given their versatility, these structures can become a useful tool in the assistance of rehabilitation work of human upper and lower limbs, and in some cases, such as this work for example, help patients with paralysis in their limbs for the development of activities as complex as human walking.

Methodology-Although there are some important advances in this area, it is still in the research phase and many of the developments are not yet available or affordable for massive use in countries such as Colombia and Latin America in general. From this point of view and as a contribution to the development of this area in the country, this work presents the development of an active exoskeleton, which was designed to assist in the rehabilitation of patients who have had some type of sequelae as a result of a Brain Vascular Accident (stroke), also known as Stroke.

Results- The article includes information on the modeling, design, control and construction of the exoskeletal device, as well as the development of preliminary tests aimed at showing its use in the development of repetition tests in the sagittal plane.

Conclusions- The result of these analyses shows the potential of the system developed for its application in this type of rehabilitation therapies.

Keywords- Exoskeleton; cerebrovascular accident; rehabilitation; lower limb; control; prototype. 


\section{INTRODUCCIÓN}

Las personas que sufren algún tipo de discapacidad, enfrentan problemas comunes que se reflejan en el detrimento de su calidad de vida, puesto que en la mayoría de los casos deben depender económicamente y socialmente de alguien para su integración en la sociedad. De acuerdo con el informe mundial sobre discapacidad, en el año 2019 [1], se estima que más de mil millones de personas viven con algún tipo de discapacidad, o sea, alrededor del 15\% de la población mundial. La Encuesta Mundial de Salud señala que del total estimado de personas con discapacidad, 110 millones $(2,2 \%)$ tienen dificultades muy significativas de funcionamiento, mientras que la Carga Mundial de Morbilidad cifra en 190 millones (3,8\%) las personas con una discapacidad grave (el equivalente a la discapacidad asociada a afecciones tales como la tetraplejía, depresión grave o ceguera) [2]. Solo la Carga Mundial de Morbilidad mide las discapacidades infantiles (014 años), con una estimación de 95 millones de niños (5,1\%), 13 millones de los cuales $(0,7 \%)$ tienen "discapacidad grave tal como se detectó desde la primera versión de este informe en el año 2011 [1].

Las personas que sufren algún tipo de discapacidad enfrentan problemas comunes que se reflejan en el deterioro de su calidad de vida, ya que en la mayoría de los casos se ven en la necesidad de depender económica y socialmente de alguien para su integración en la sociedad. De acuerdo al informe mundial de discapacidad, publicado en 2011 y los datos del banco mundial en 2019 [1], alrededor del 15\% de la población mundial viven con algún tipo de discapacidad. Dentro de la encuesta mundial de salud se estima que dentro de la población mayor de 15 años, el $15.6 \%$ padece algún tipo de discapacidad y de estas alrededor del $2.2 \%$ presentan gravedad en su discapacidad [3].

En Colombia desde el año 2000 mediante el convenio de cooperación 025 suscrito entre el Ministerio de Comunicaciones y la Organización de Estados Iberoamericanos, se empieza a trabajar sobre el marco teórico del sistema para medir la discapacidad en el territorio, basándose en un formulario único como instrumento para la recolección de información, el cual está fundamentado en el marco conceptual de Clasificación Internacional del Funcionamiento, la Discapacidad y la Salud (CIF) de la Organización Mundial de la Salud [4]. De la misma manera, el DANE como entidad responsable de la información estadística del país, firmó un convenio con el Ministerio de Educación Nacional (MEN) dirigido a la "implementación del Registro para la Localización y Caracterización de las personas con discapacidad, RLCPD” en cuyo informe para el segundo semestre del 2018 se indica que el $2.6 \%$ de la población padece de alguna discapacidad, es decir alrededor de 1.418.065 personas, de las cuales el $58 \%$ son mayores de 50 años y el $12 \%$ son menores de edad [6]; en este informe también se indica que el $22 \%$ de las personas con alguna discapacidad necesitan rehabilitación con fisioterapia, teniendo en cuenta que la discapacidad que se presenta con mayor frecuencia en la población es el Movimiento del cuerpo, manos , brazos y piernas con un indicador de $34,2 \%$. Esta cifra refleja que poco más de trecientas once mil personas requieren de atención y/o soporte médico para superar estas situaciones físicas, mostrando un problema en el que diversas profesiones pueden aportar soluciones de manera multidisciplinar, por otra parte, uno de los factores que tiene gran incidencia en la población colombiana, es el creciente desarrollo de enfermedades cerebrovasculares que día a día ganan un mayor terreno, convirtiéndose en un problemática de salud pública en Colombia, este factor se consolida como la cuarta causa de muerte en la población adulta mayor y representa un factor de alta discapacidad entre los pacientes que logran sobrevivir a estos casos [5].

Como contribución a solucionar este problema, en el presente artículo se describe el desarrollo de un exoesqueleto para rehabilitación basado en el paradigma de rehabilitación de miembros inferiores basado en terapias de repeticiones en pacientes con ictus. Este artículo se encuentra organizado en siete secciones que incluyen esta introducción, un breve estado del arte de exoesqueletos en la sección 2 , un marco teórico que incluye información sobre la terminología empleada en la sección 3. Sección 4, describe el sistema de control desarrollado, mientras que la sección 5 describe el exoesqueleto desarrollado junto con las pruebas de funcionamiento del sistema. Finalmente, la sección 6 incluye las conclusiones del trabajo.

\section{EstAdo DEL ARTE}

La Organización Mundial de la Salud (OMS), define la enfermedad cerebrovascular como el rápido desarrollo de signos focales o globales de compromiso de la función cerebral [7]. La relevancia que tienen las patologías que causan ciertas enfermedades de tipo cerebrovascular, se funda en su alta prevalencia y el impacto que tiene sobre la calidad de vida de las personas, consecuencia que se refleja en una discapacidad permanente y su alto índice de mortalidad [8], [9]. Actualmente se han desarrollado algunos trabajos para dar solución a los diversos problemas de movilidad de los miembros inferiores, entre ellos por ejemplo el desarrollo de sistemas para asistencia en marcha [10], tratamiento de discapacidades motoras debido a parálisis cerebral [11] y desarrollo de aplicaciones de control para sistemas exoesqueléticos [12]. Sin embargo, los primeros modelos de exoesqueletos aparecieron en el año de 1960 en los laboratorios de la Universidad de Cornell [8] [43]. 
La estructura de Yang es uno de los primeros intentos registrados con características exo-esqueléticas, la cual tenía por objetivo primordial aumentar la potencia de ritmo de caminata sí como de los saltos [13]. Otra versión de este tipo de exoesqueleto fue implementada con motores de corriente continua, aunque el motor, el sistema de alimentación (batería) y la tecnología computacional del momento, limitaban en alto grado la portabilidad del dispositivo [14]. Uno de los modelos más exitosos para la rehabilitación de pacientes parapléjicos y personas con discapacidad similares, se desarrolló en el año de 1972 en el Instituto Central de Traumatología y Ortopedia en Moscú, en el marco de cooperación científica URSS-Yugoslavia. Este diseño contaba con partes electromecánicas, programado electrónicamente, y fue probado en 1974. Este fue uno de los primeros diseños donde se utilizó motores como actuadores para generar movimiento [13]. Otro de los trabajos más importantes fue presentado en 1981 por Jaukovic en la Universidad de Titograd en la antigua Yugoslavia, este dispositivo estaba construido a partir de motores en dc permitiendo así la flexión y extensión del tobillo, en este trabajo se incluyeron sensores ubicados en la planta del pie. El desarrollo del prototipo estaba enfocado hacia la rehabilitación de personas que habían padecido algún accidente cerebrovascular o parálisis cerebral [14].

Entre los últimos modelos más destacados se encuentra BLEEX, resultado del sistema DARPA implementado por la Universidad de Berkeley en California (USA) [15], el cual se caracteriza por contar con un sistema de carga autónomo, con 3 grados de libertad en la cadera, 1 en la rodilla y 3 en el tobillo, de los cuales 4 son accionados de forma: cadera, (flexión / extensión), (hip abducción / aducción), en la flexión de la rodilla (flexión / extensión), y en el tobillo (flexión / extensión). Al igual que un robot bípedo, el exoesqueleto puede equilibrarse por sí solo, pero el sujeto debe proporcionar el ritmo guiando la fuerza para dirigir el sistema durante la marcha [16].

Otro tipo de exoesqueleto conocido con el nombre de "Lopes" [17], es un sistema utilizado en pacientes para el reentrenamiento de la marcha y su principal característica se fundamenta en el sistema de control, el cual se apoya en un entorno virtual con el objetivo de establecer un vínculo más directo entre el sistema robótico y el usuario, asegurando un patrón de marcha pre-programado [18].

Otro de los modelos representativos de exoesqueletos se diseñó bajo quince figuras geométricas simples [19], [28], [29], las cuales conformaban el cuerpo humano usando el modelo de Hanavan. Hacen uso del programa Screws para representar el entorno físico del robot y de los posibles movimientos, considerándose hasta siete grados de libertad. Para obtener la cinemática en este exoesqueleto, se utilizó el algoritmo de denavit-hartenberg.
Recientes modelos implementados son encaminados a ambientes clínicos, como es el caso del modelo diseñado por Universidad Tecnológica de Berlín (Berlin, Alemania), un tipo de ortesis de alta potencia, donde las señales electromiográficas registradas en cada uno de los músculos, sirve como control de torque al motor encargado de realizar el movimiento de la rodilla [20].

En la Universidad Politécnica de Catalunya (Barcelona, España), se presenta un diseño de ortesis activa rodilla-tobillo [21], la cual se enfoca en la asistencia y recuperación de pacientes con lesión medular, ya que su condición tiende a dejarlos sin movilidad casi en un 80\% de su cuerpo.

Vanderbilt es un prototipo de exoesqueleto desarrollado en la Universidad de Vanderbilt (Tennesssee, USA), con cerca de $12 \mathrm{Kg}$ de peso, cuenta con dispositivos electrónicos en las articulaciones del sujeto. El dispositivo es alimentado por motores dc con un diseño de engranajes el cual aumenta la fuerza proporcionando un torque cercano a los $12 \mathrm{~N}$ para la cadera, rodilla y tobillo respectivamente; el prototipo es usado en diferentes pruebas bajo supervisión médica en el hospital de Atlanta (USA), en pacientes que presentan antecedentes de lesión de la médula espinal [22], [23]. El desarrollo de un exoesqueleto de cadera es presentado en [42], el cual incluye alta potencia, autobalance y un software para realizar el control del movimiento y mantener el balance en el plano sagital y el frontal. Este exoesqueleto incluye los movimientos de abducción y aducción de cadera, así como flexión y extensión de la misma. Con el fin de tener mayor estabilidad en los movimientos, se desarrolla un controlador basado en el concepto de centro de masa extrapolado. El prototipo es evaluado en una persona sana mostrando la efectividad del desarrollo y el controlador diseñado.

Existen así mismos desarrollos de exoesqueletos enfocados hacia recuperación de pacientes que presentan accidentes cerebrovasculares [41], otros trabajos relacionados con el estado del arte de los exoesqueletos [43], muestran con mayor detalle los avances en la tecnología usada en este campo, los grandes beneficios de este tipo de desarrollos y el trabajo a futuro en este campo.

A nivel de Latinoamérica existen desarrollos de exoesqueletos para rehabilitación de brazo y pierna. En cuanto a rehabilitación de brazo, por ejemplo, se tienen desarrollos con cuatro grados de libertad orientados a la antropometría de pacientes mexicanos usando diseño conceptual óptimo [36], exoesqueletos orientados al movimiento de rotación del húmero, flexión y extensión del codo [39], pronación y supinación de la mano [38]. En el caso de rehabilitación en pierna se tienen desarrollos de dos grados de libertad para terapias en tobillo y rodilla, incluyendo uso de sensores de fuerza, velocidad y posición [37]. Así como diseños exclusivos para flexión-extensión de rodilla [40]. 
Por parte de los autores, el desarrollo de actividades en el área de exoesqueletos inició en el año 2007 con la dirección de una tesis de pregrado del ingeniero Juan Grosso en la Universidad Autónoma de Bucaramanga (Colombia). En este trabajo se realizó el estudio de varios modelos biomecánicos y se orientó la definición de un primer modelo de exoesqueleto basado en el uso y ajuste de un modelo biomecánico para un paciente con unas características definidas [29], [31]. A partir de este trabajo y otros posteriores, se llegó a una implementación a escala de un exoesqueleto que permitió validar la cinemática y la dinámica de este sistema pero que no incluía el desarrollo a escala real ni el diseño ajustable a diversos pacientes. Posterior a estos desarrollos, se trabajó en la generalización del modelo biomecánico y en el análisis paramétrico del sistema para determinar la influencia de los parámetros en la cinemática y la dinámica del exoesqueleto para dos grados de libertad [28], [30], [35]. Otros trabajos de los autores, incluyen simulaciones de la cinemática de exoesqueletos para brazo humano considerando robots de tipo serial y paralelo [32], [33], [34]. A diferencia de los trabajos anteriores, el exoesqueleto presentado en este artículo se enfoca a rehabilitación de personas con accidentes cerebrovasculares, e incluye una implementación física producto del diseño mecatrónico para movimientos en el plano sagital.

\section{MARCO TEÓRICO}

En el desarrollo del exoesqueleto propuesto en este trabajo, se manejaron diferentes conceptos relacionados con el modelo biomecánico del cuerpo humano y la cinemática de los manipuladores que incluye algoritmo de Denavit-Hartenberg aplicado al miembro inferior.

\section{A. Modelo biomecánico}

Es importante a la hora de realizar el diseño del exoesqueleto, determinar el rango del movimiento a realizar, y para este caso se trabaja el miembro inferior, el cual consta de tres componentes (cadera, pierna y pie) (Fig. 1).

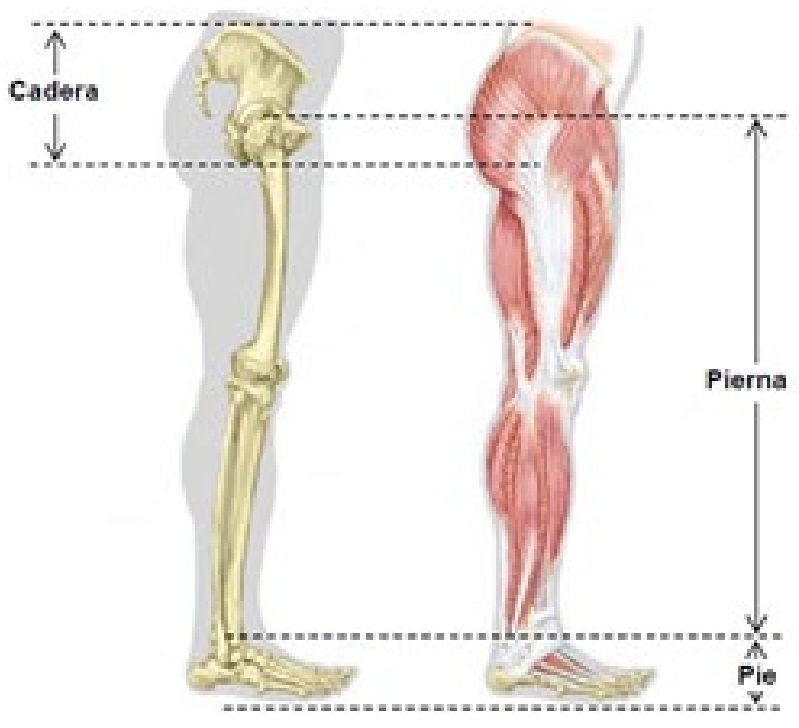

Fig. 1. Anatomía del miembro inferior Fuente: Autores.
Los movimientos en el miembro inferior se enfocan en la pierna y la rodilla. La pierna puede llegar a presentar un hiperextensión de $15^{\circ}$ grados, mientras que la flexión máxima de la misma alcanza un rango de $130^{\circ}$ a $140^{\circ}$ grados. Por otra la hiperextensión en la rodilla alcanza $-10^{\circ}$ grados y la flexión máxima $155^{\circ}$ grados (cuando el talón toca el glúteo) [8].

Los modelos biomecánicos intentan representar de forma matemática la estructura y las capacidades del cuerpo humano que se relacionan con la movilidad del mismo. Dentro de estos modelos se pueden encontrar con tres representantes como son el modelo Harless, Hanavan y Drillis y Contini, en la tabla 1 se muestra una breve descripción de cada modelo.

Tabla 1. Modelos Biomecánicos.

\begin{tabular}{|c|l|}
\hline $\begin{array}{c}\text { Modelo } \\
\text { de } \\
\text { Harlless }\end{array}$ & $\begin{array}{l}\text { Este modelo se enfoca en el cálculo del volumen } \\
\text { de cada parte del cuerpo utilizando el método de } \\
\text { inmersión. Como resultado se obtienen los centros } \\
\text { de masa de cada parte del cuerpo. }\end{array}$ \\
\hline $\begin{array}{c}\text { Modelo } \\
\text { de }\end{array}$ & $\begin{array}{l}\text { En este modelo matemático se estudian } \\
\text { los parámetros inerciales partiendo de una } \\
\text { segmentación del cuerpo en 15 partes las cuales se } \\
\text { Hanavan }\end{array}$ \\
$\begin{array}{l}\text { representan geométricamente mediante cilindros. } \\
\text { gomo resultado se obtienen medidas del centro de } \\
\text { gravedad, los momentos y productos de inercia. }\end{array}$ \\
\hline $\begin{array}{c}\text { Este modelo se basa en un estudio estadístico } \\
\text { en donde a partir del peso total y estatura de } \\
\text { Drillis y } \\
\text { Continis }\end{array}$ & $\begin{array}{l}\text { una persona se estima el peso y longitud de los } \\
\text { del modelo tienen un error inferior a 1cm. }\end{array}$ \\
\hline
\end{tabular}

Fuente: [9], [28].

\section{B. Modelado del exoesqueleto}

Es posible realizar el análisis de la pierna humana considerando el sistema de doble péndulo invertido como un mecanismo de dos grados de libertad integrado entre sí mediante una sola articulación; teniendo en cuenta que el comportamiento del péndulo invertido y el miembro inferior son semejantes siempre y cuando se conserve las restricciones anatómicas de rotación, se considera el sistema inicial tal como el mostrado en la Fig. 2, el cual se moverá alrededor de los ejes Y0 y Z0, incluyendo actuadores mecánicos en las articulaciones.

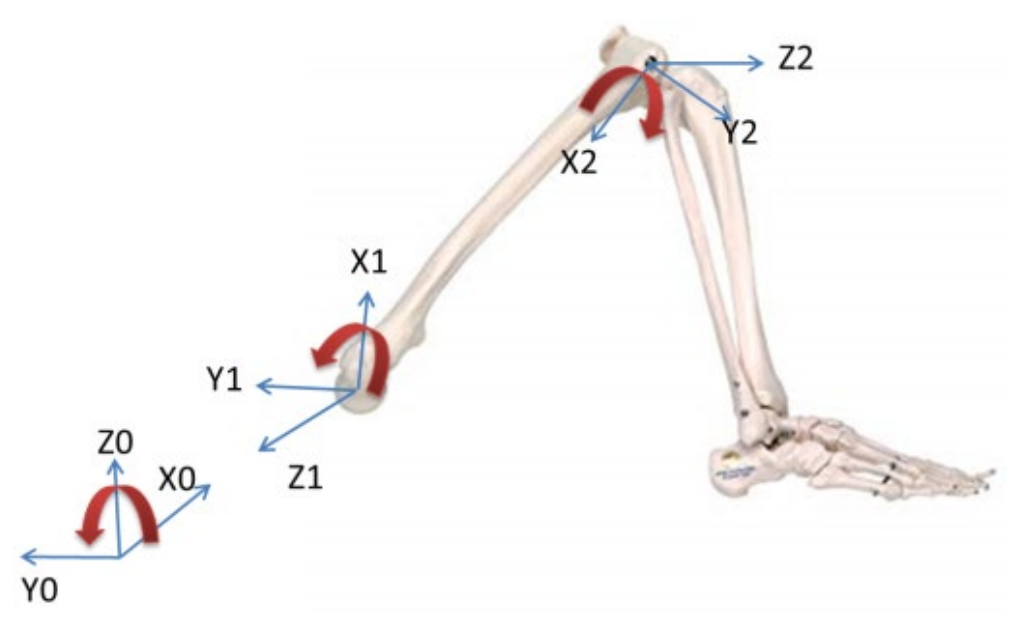

Fig. 2. Sistema de coordenadas. Fuente: Autores. 
El estudio de la posición, velocidad y aceleración que presenta el sistema se realiza utilizando la teoría de cinemática de manipuladores, en esta se calculan las matrices homogéneas que permitirán describir la cinemática directa de la pierna (1) considerando los parámetros del algoritmo de Denavit-Hartenberg definidos en la Tabla 2 y mostrados en el robot de dos grados de libertad de la Fig. 3.

$$
\left(\begin{array}{c}
X_{0} \\
Y_{0} \\
Z_{0}
\end{array}\right)=\left(\begin{array}{c}
l_{2} \cos \left(q_{1}+q_{2}\right)+l_{1} \cos \left(q_{1}\right) \\
l_{2} \sin \left(q_{1}+q_{2}\right)+\sin \left(q_{1}\right) \\
B \quad B_{2}
\end{array}\right)
$$

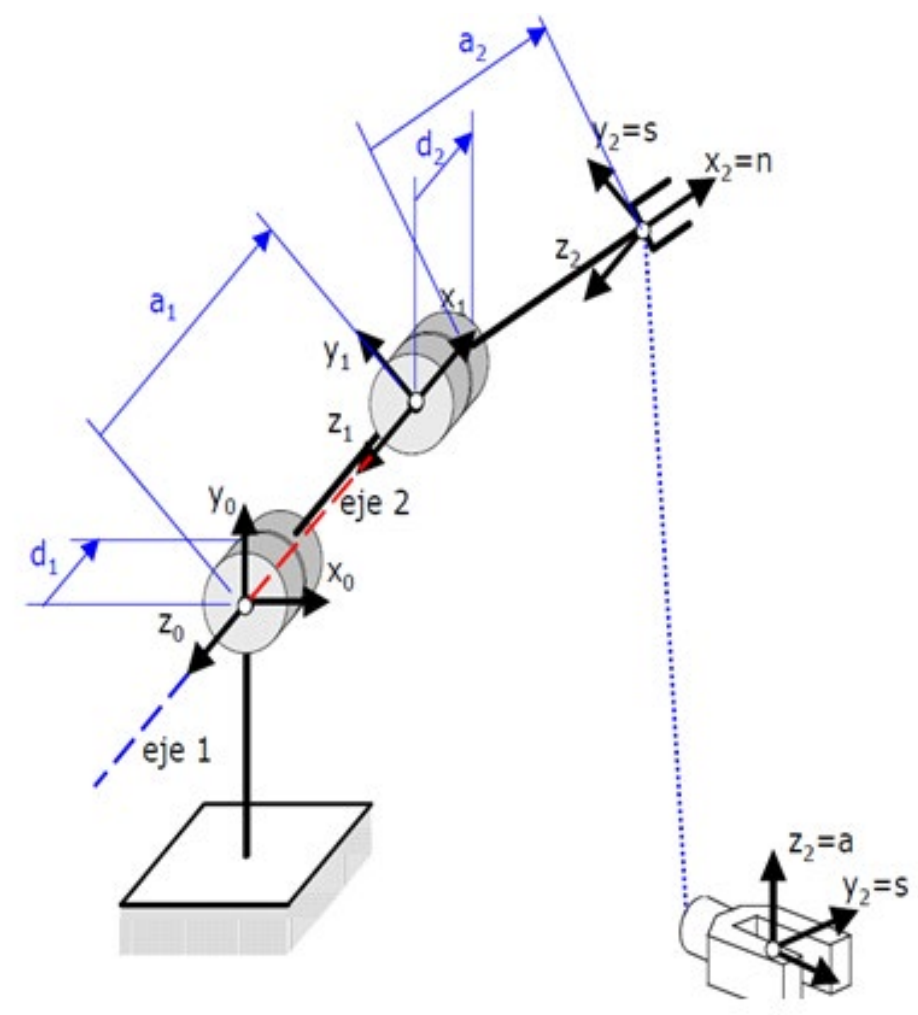

Fig. 3. Sistema de coordenadas en miembro inferior

Fuente: Autores.

Tabla 2. Parámetros Denavit-Hartenberg

\begin{tabular}{|c|c|c|c|c|}
\hline Articulación & $\mathbf{a}_{\mathbf{i}}$ & $\mathrm{a}$ & $\mathbf{D}$ & $\boldsymbol{\Theta}$ \\
\hline 1 & $\mathrm{~L}_{1}$ & 0 & $\mathrm{~d}_{1}$ & $\mathrm{q}_{1}{ }^{*}$ \\
\hline 2 & $\mathrm{~L}_{2}$ & 0 & $\mathrm{~d}_{2}$ & $\mathrm{q}_{2}{ }^{*}$ \\
\hline
\end{tabular}

Para la dinámica se utilizó la formulación lagrangiana, en esta el modelo está determinado por la diferencia entre la energía cinética y la energía potencial tal como se muestra en (2).

$$
L(q(t), \dot{q}(t))=K(q(t), \dot{q}(t))-U(q(t))
$$

Para el caso de dos grados de libertad, como en el caso del exoesqueleto desarrollado, las energías cinética y potencial quedan en función de cada eslabón como se muestra (3) y (4).

$$
\begin{aligned}
& K(q, \dot{q})=K_{1}(q, \dot{q})+K_{2}(q, \dot{q}) \\
& U(q)=U_{1}(q)+U_{2}(q)
\end{aligned}
$$

Los parámetros de cada eslabón se incluyen en la Tabla 3, de acuerdo a la Fig. 4. Cada uno de los parámetros fueron calculados considerando el modelo de Hanavan, el cual considera al cuerpo divido en secciones tal como se muestra en la Fig. 5. Y el modelo de Drillis y Contini, el cual describe entre otras, la longitud de los segmentos del cuerpo humano.

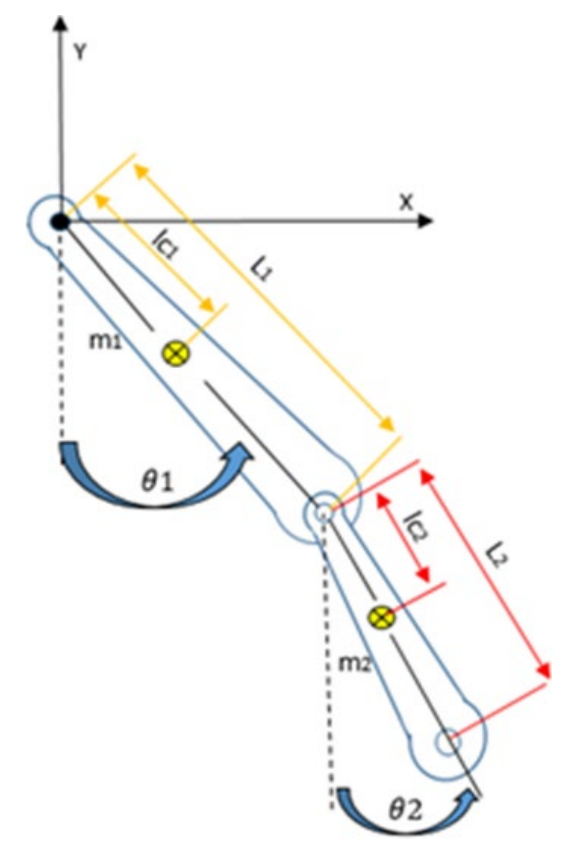

\begin{tabular}{|c|c|c|c|}
\hline Eslabón & Descripción & Notación & Cantidad \\
\hline \multirow{7}{*}{$\begin{array}{l}\text { Articulación } \\
1 \text { (muslo) }\end{array}$} & Masa articulación 1 & M1 & $8 \mathrm{~kg}$ \\
\hline & Longitud eslabón 1 & L1 & $0.57 \mathrm{~m}$ \\
\hline & Inercia del eslabón 1 & I1 & $\begin{array}{l}2.59 \mathrm{Nm} \\
\mathrm{seg}^{2} / \mathrm{rad}\end{array}$ \\
\hline & $\begin{array}{l}\text { Centro de masa } \\
\text { eslabón } 1\end{array}$ & Lc1 & $0.23 \mathrm{~m}$ \\
\hline & $\begin{array}{l}\text { Coeficiente de } \\
\text { fricción viscosa }\end{array}$ & b1 & $\begin{array}{c}2.288 \mathrm{Nm} \\
\mathrm{seg} / \mathrm{rad}\end{array}$ \\
\hline & $\begin{array}{c}\text { Coeficiente de } \\
\text { fricción de coulomb }\end{array}$ & fc1 & $7,17 \mathrm{Nm}$ \\
\hline & $\begin{array}{l}\text { Coeficiente de } \\
\text { fricción estática }\end{array}$ & fe1 & $8.8 \mathrm{Nm}$ \\
\hline \multirow{8}{*}{$\begin{array}{l}\text { Articulación } \\
2 \text { (pierna) }\end{array}$} & Masa articulación 2 & M2 & $4 \mathrm{~kg}$ \\
\hline & Longitud eslabón 2 & L2 & $0.45 \mathrm{~m}$ \\
\hline & Inercia del eslabón 2 & $\mathrm{I} 2$ & $\begin{array}{c}0.81 \mathrm{Nm} \\
\mathrm{seg}^{2} / \mathrm{rad}\end{array}$ \\
\hline & $\begin{array}{l}\text { Centro de masa } \\
\text { eslabón } 2\end{array}$ & lc2 & $0.21 \mathrm{~m}$ \\
\hline & $\begin{array}{c}\text { Coeficiente de } \\
\text { fricción viscosa }\end{array}$ & b2 & $\begin{array}{c}0.175 \mathrm{Nm} \\
\mathrm{seg} / \mathrm{rad}\end{array}$ \\
\hline & $\begin{array}{l}\text { Coeficiente de } \\
\text { fricción de coulomb }\end{array}$ & $\mathrm{fc} 2$ & $1,734 \mathrm{Nm}$ \\
\hline & $\begin{array}{l}\text { Coeficiente de } \\
\text { fricción estática }\end{array}$ & fe2 & $1.87 \mathrm{Nm}$ \\
\hline & $\begin{array}{l}\text { Aceleración debida a } \\
\text { la gravedad }\end{array}$ & $\mathrm{g}$ & $9.8 \mathrm{~m} / \mathrm{s}^{2}$ \\
\hline
\end{tabular}

Fig. 4. Descripción del sistema de dos eslabones Fuente: Autores.

Tabla 3. Parámetros de Cada eslabón.

Fuente: Autores. 


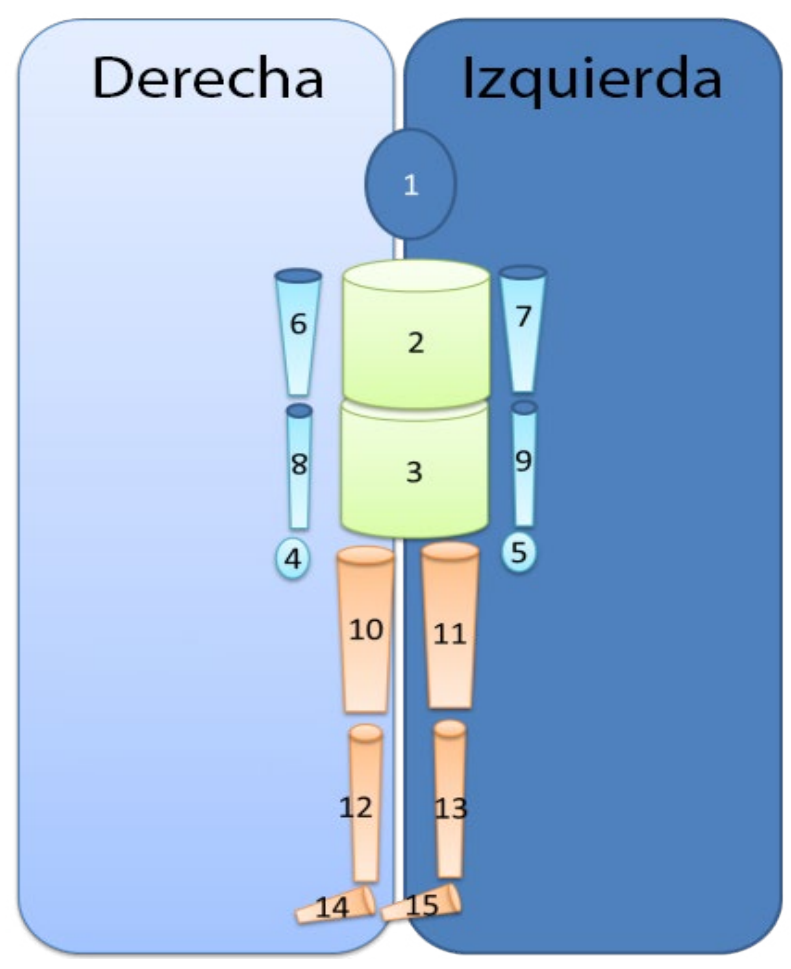

Fig. 5. Modelo de Hanavan.

Fuente: Autores.

De acuerdo con la Tabla 3, el modelo matemático final del sistema (5) y (6) en función de la dinámica, utiliza las ecuaciones del movimiento de Euler-Lagrange [24], teniendo como referencia el esquema de junturas, eslabones y ejes coordenados mostrados (Fig. 4). Esta forma de modelar es de gran ayuda para determinar la relación de todos los parámetros inmersos que se encuentran en el modelo del exoesqueleto [25].

$$
\begin{gathered}
\tau_{1}=\left[2 a \cos \left(\theta_{2}\right)+m_{1} L_{C_{1}}^{2}+m_{2} L_{1}^{2}+b\right] \ddot{\theta}_{1}+ \\
{\left[I_{1}+I_{2}\right] \ddot{\theta}_{1}+\left[a \cos \left(\theta_{2}\right)+b+I_{2}\right] \ddot{\theta}_{2}-} \\
2 a \sin \left(\theta_{2}\right) \dot{\theta}_{1} \dot{\theta}_{2}-a \sin \left(\theta_{2}\right) \dot{\theta}_{2}^{2}+\left[m_{1} L_{c_{1}}+\right. \\
\left.m_{2} L_{1}\right] g \sin \left(\theta_{1}\right)+c\left(g \sin \left(\theta_{1}+\theta_{2}\right)\right) \\
\tau_{2}=\left[a \cos \left(\theta_{2}\right)+b+I_{2}\right] \theta_{1}+\left[b+I_{2}\right] \\
\ddot{\theta}_{2}+a \sin \left(\theta_{2}\right) \dot{\theta}_{1}^{2}+c\left(g \sin \left(\theta_{1}+\theta_{2}\right)\right)
\end{gathered}
$$

\section{SISTEMA \\ EXOESQUELÉTICO ACTIVO}

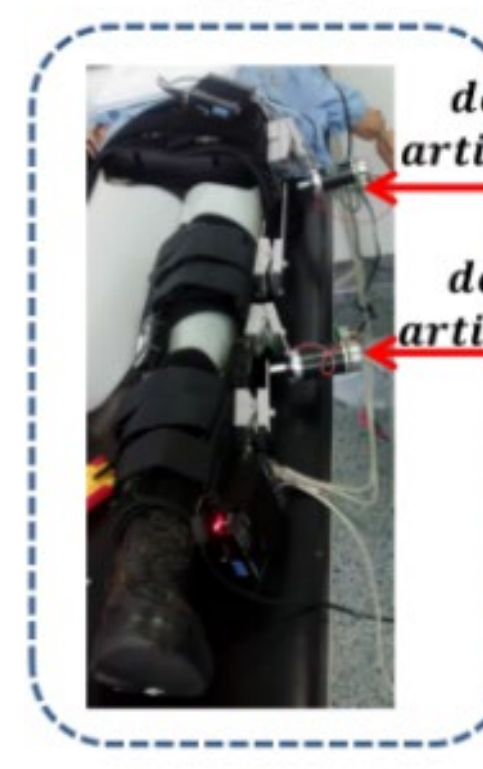

- señal deicontrol artidulación 1 \eñal deicontrol articulación 2

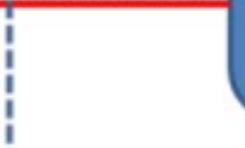
$\theta_{1}$ actua $\theta_{2}$ actual

$$
\dot{X}=(A+\Delta)+X+B t
$$

$$
\theta=C X(t)+D(t)
$$

Fig. 6. Sistema de control

Fuente: Autores. 
Para el exoesqueleto, se considera el control de posición definido por una trayectoria previamente establecida para el proceso de terapia. Esta trayectoria será capturada del mismo exoesqueleto mediante sensores de tipo encoder acoplados a cada uno de los motores, los cuales brindan información de la posición por unidad de tiempo. Esto es realizado en una primera instancia por el fisioterapeuta que aplicará la terapia y una vez capturada la información, el software desarrollado permite que se pueda definir el número de repeticiones y las diferentes terapias que debe ejecutar el exoesqueleto. El seguimiento de la trayectoria que el exoesqueleto debe realizar, está a cargo del sistema de control ya que es un sistema multivariable, en el cual se tiene en cuenta los ángulos de la cadera y de la rodilla por medio de torques de entrada en cada una de ellas. En la Fig. 6 se describe la estructura de control del sistema junto con sus entradas y respectivas salidas. Para ello se contó con el driver de control fabricado por Maxon Motors y el cual fue utilizado para el control del movimiento de cada uno de los eslabones del exoesqueleto. Este controlador permite un control de posición en términos de velocidad y control de corriente. Gracias a las características técnicas con las que cuenta, se puede configurar el control de aceleración y de frenado los cuales se pueden ajustar a partir de las librerías disponibles por maxon [26].

El control de posición epos2 esta implementado bajo el estándar PID [26], donde la compensación de realimentación proporciona un ajuste más rápido en aplicaciones con mayor inercia de carga y aceleraciones o en aplicaciones mecatrónicas con una carga considerable. En la Fig. 7, se observa el comportamiento de la posición angular para el sistema de exoesqueleto desarrollado, se aprecia que los ángulos Q1 y Q2 siguen la referencia indicada.

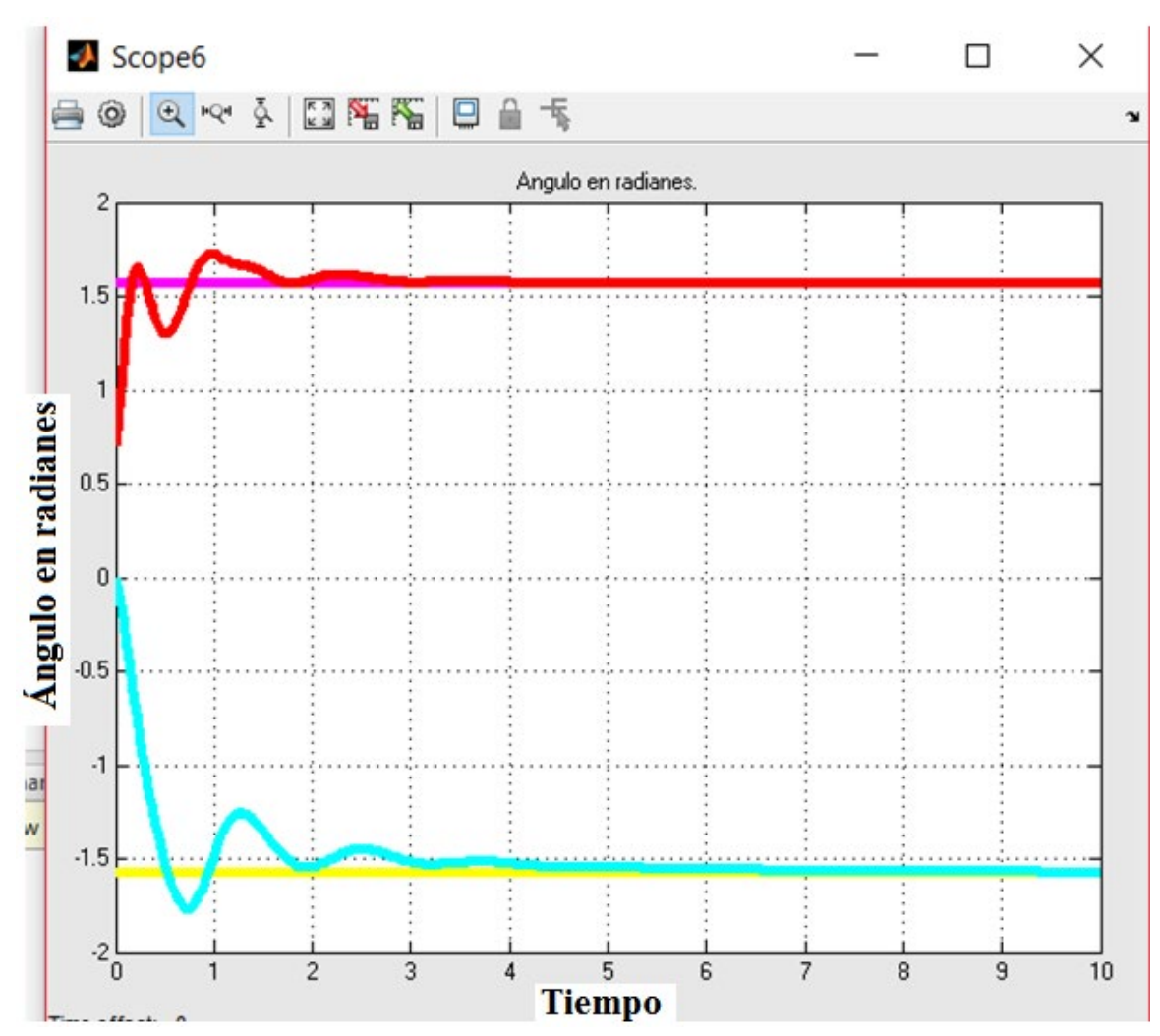

Fig. 7. Respuesta del controlador PID con realimentación de velocidad diseñado.

Fuente: Autores.

Tal como se observa (Fig. 7), el control permite que el tiempo de establecimiento sea inferior a 2 segundos con un sobreimpulso pequeño, lo cual como se muestra en la siguiente sección no perjudica el desarrollo de la terapia ni produce efectos adversos perceptibles por el usuario.

\section{Exoesqueleto DESARRollado}

La Fig. 8 y Fig. 9 muestran el exoesqueleto desarrollado y la Tabla 5 las características de movimiento para las articulaciones consideradas en el sistema. Este cuenta con un diseño de sistema deslizante en cada una de las articulaciones, lo que sirve para ajustar de manera sencilla las diferentes longitudes para diferentes pacientes. Asimismo el diseño presente en

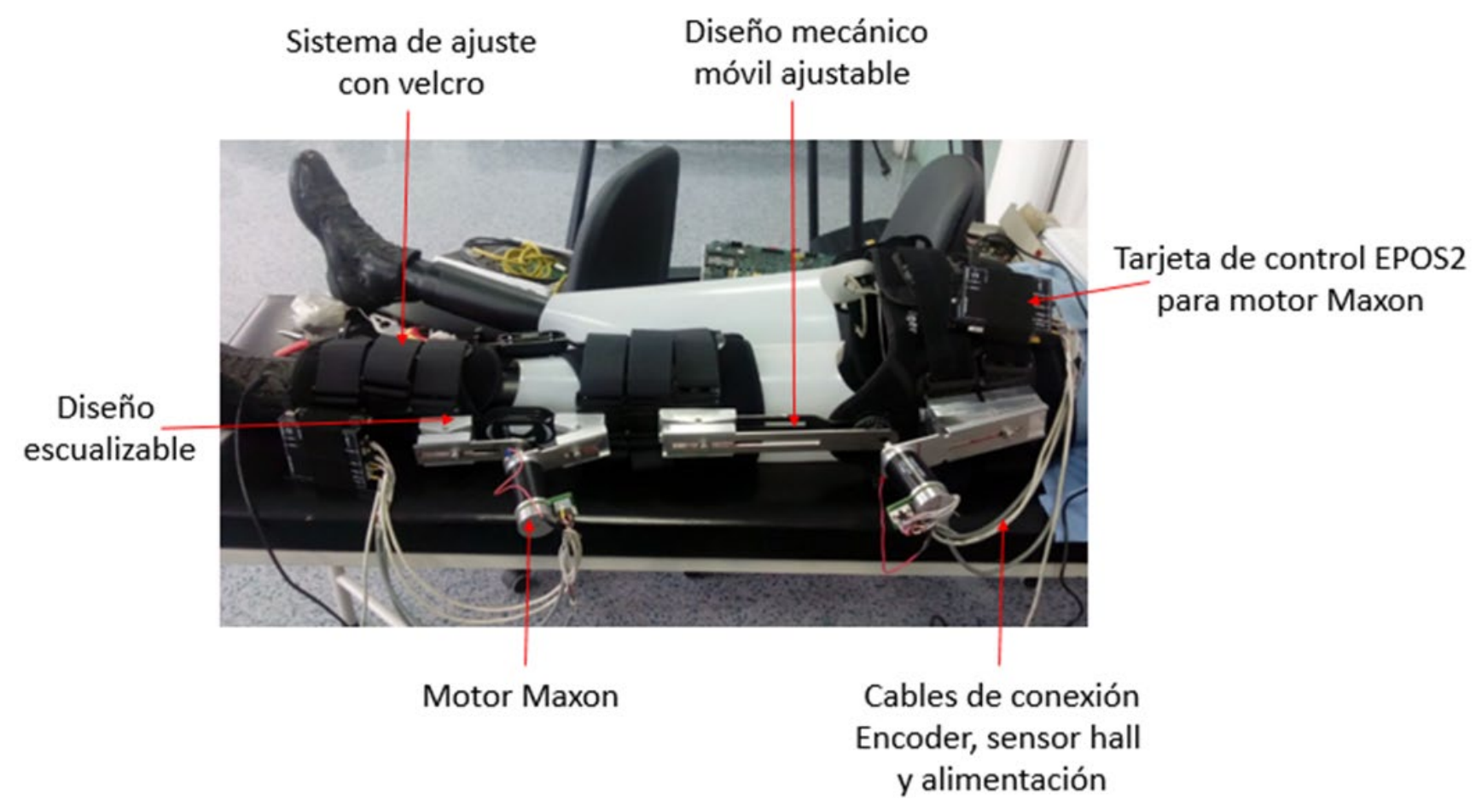

Fig. 8. Exoesqueleto desarrollado.

Fuente: [8] 
cada una de las articulaciones, está pensado para cada uno de los ejes de giro se comporte de manera escualizable, el cual tiene el propósito de buscar una adaptación optima y la mejor ergonomía de manera natural al paciente.

TABla 5. Características de MOVImiento DEL EXOESQUELETO DESARROLLADO.

\begin{tabular}{|c|c|c|c|}
\hline Eje de rotación & Movimiento & Máximo (grados) & Plano \\
\hline \multirow{2}{*}{ Cadera } & Flexión & 145 & sagital \\
\cline { 2 - 4 } & Extensión & 30 & sagital \\
\hline \multirow{2}{*}{ Rodilla } & Flexión & 10 & sagital \\
\cline { 2 - 4 } & Extensión & 120 & sagital \\
\hline
\end{tabular}

Fuente: Autores.

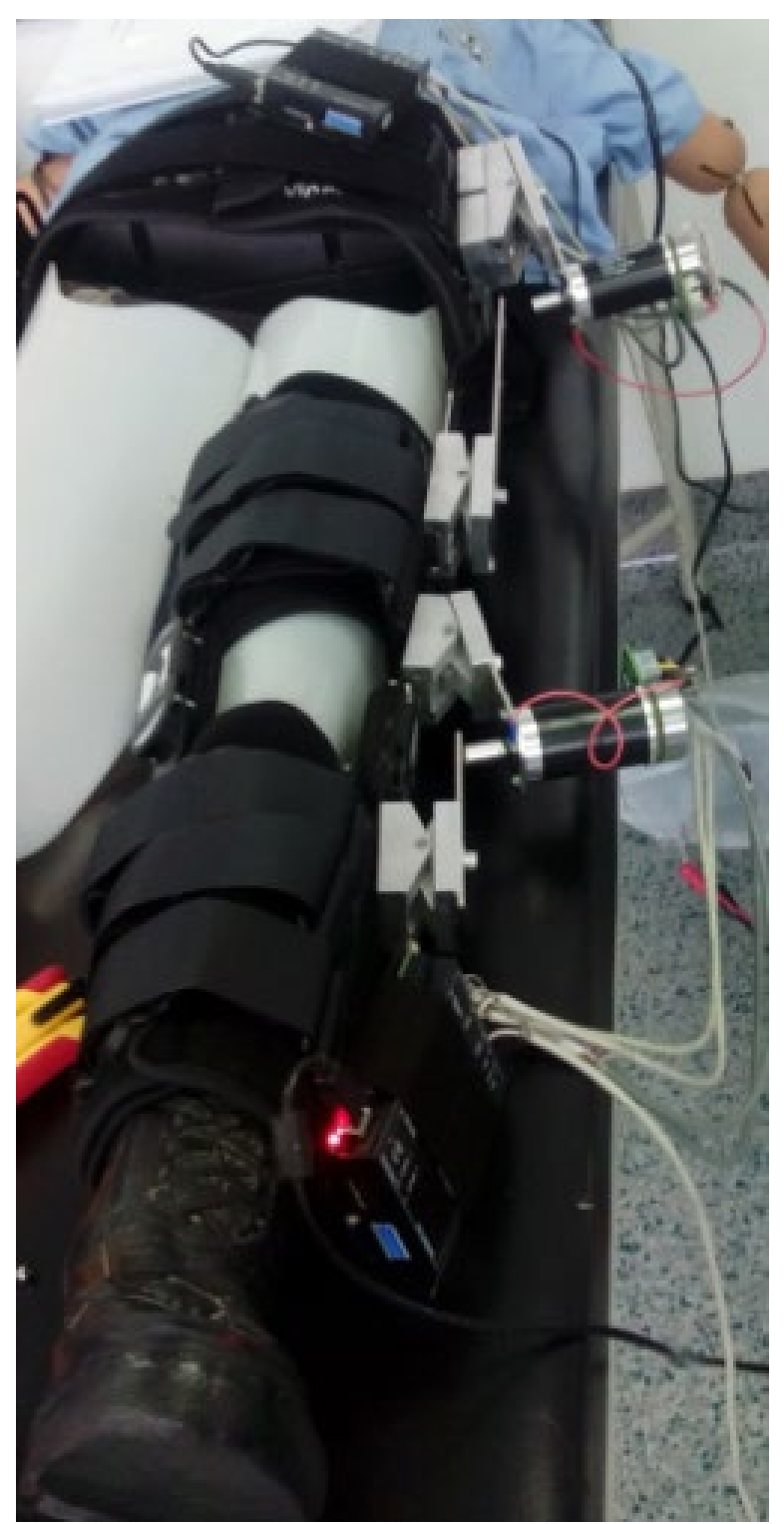

Fig. 9. Exoesqueleto desarrollado y plataforma para pruebas. Fuente: [9]

Los actuadores utilizados fueron dos motores maxon EC 45 Flat que se acoplan al exoesqueleto tal como se muestra en la Fig. 9 y son los encargados de realizar el movimiento de las articulaciones, a los motores se acoplan los transductores rotativos conocidos como encoders, de los cuales se recoge la información de posición del eje del motor [27]; estos motores incluyen un sistema de reducción que permite incrementar el torque aplicado a la estructura. La Fig. 10 muestra el acople desarrollado para ajustar el exoesqueleto a la camilla, de tal manera que se pueda evitar oscilaciones del motor que puedan ser perjudiciales para el desarrollo de la terapia. El valor aproximado en pesos colombianos del prototipo es de 9 millones de pesos, sin embargo este valor puede cambiar en una versión final y si se considera el desarrollo del dispositivo en masa, lo que abarataría el sistema.

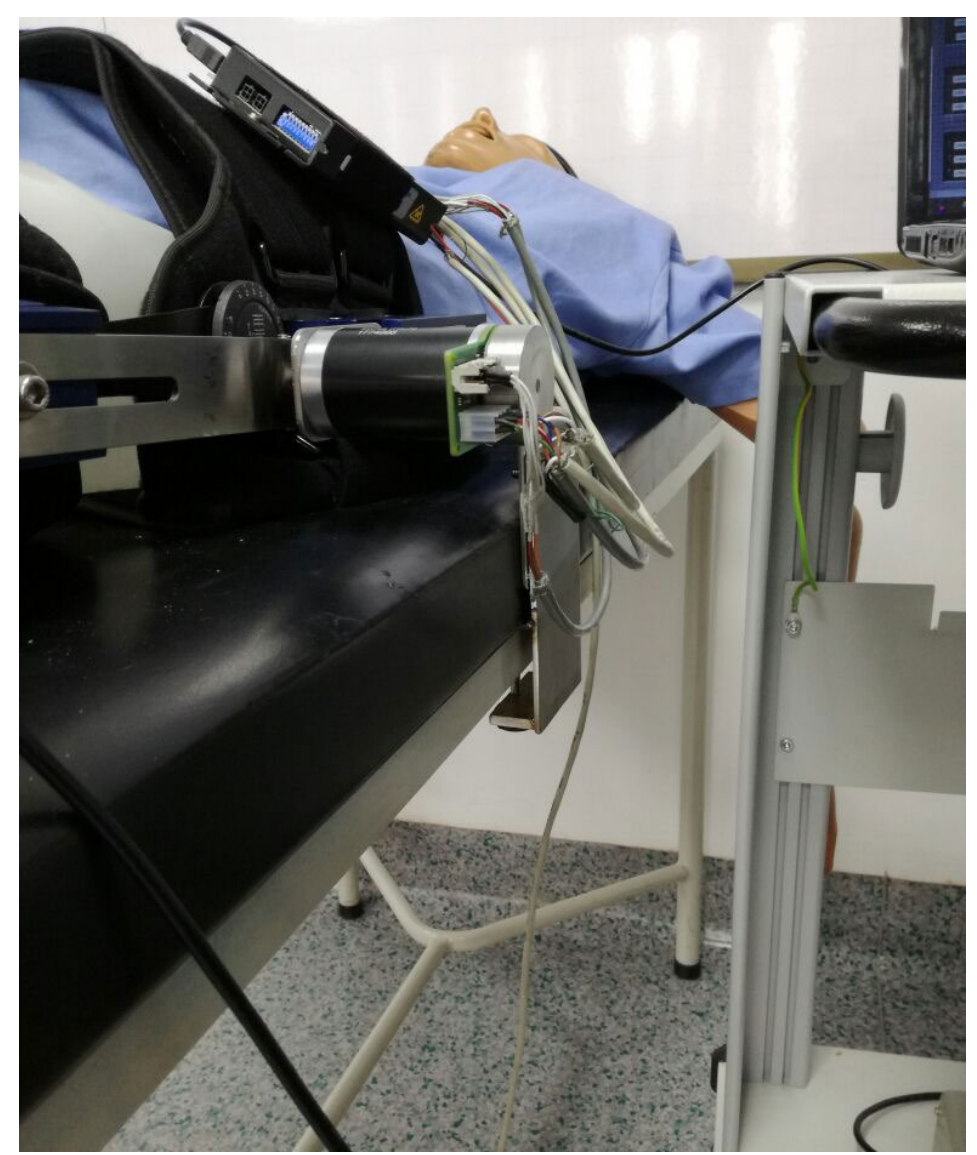

Fig. 10. Soporte de cadera acoplado a la camilla.

Fuente: [9]

Con el fin de manejar de la mejor manera el control de posición del exoesqueleto, se desarrolló una interfaz (Fig. 11) a partir del driver Epos2; el objetivo de esta es poder configurar el ángulo de movimiento (InicialFinal) de cada uno de los eslabones.

La interfaz se compone de tres fases o etapas, dentro de las que se tienen la configuración de los actuadores, selección del set point de cada motor y la visualización de la señal de control. En la primera etapa, se configuran los actuadores en términos de velocidad, aceleración, y desaceleración (o tiempo de frenado). En la segunda etapa, se seleccionan los grados de movimiento que se desea programar al motor, teniendo presente los rangos de movimiento presentados en la Tabla 5. Por último, se tiene la etapa de visualización del comportamiento de las señales de control, en este caso son la posición, la velocidad y la corriente.

Una vez configurada cada una de las etapas mencionadas anteriormente, la interfaz permite ingresar el número de veces que se desea realizar la repetición del movimiento y finalmente queda a la espera de confirmar el inicio de la terapia a través de un botón de inicio.

Para validar el exoesqueleto desarrollado, se realizan pruebas de repetición con el actuador, en donde se verifica el comportamiento del actuador ante un set point (posición) deseado, su capacidad para mantener el mismo y el tiempo necesario para alcanzarlo. 


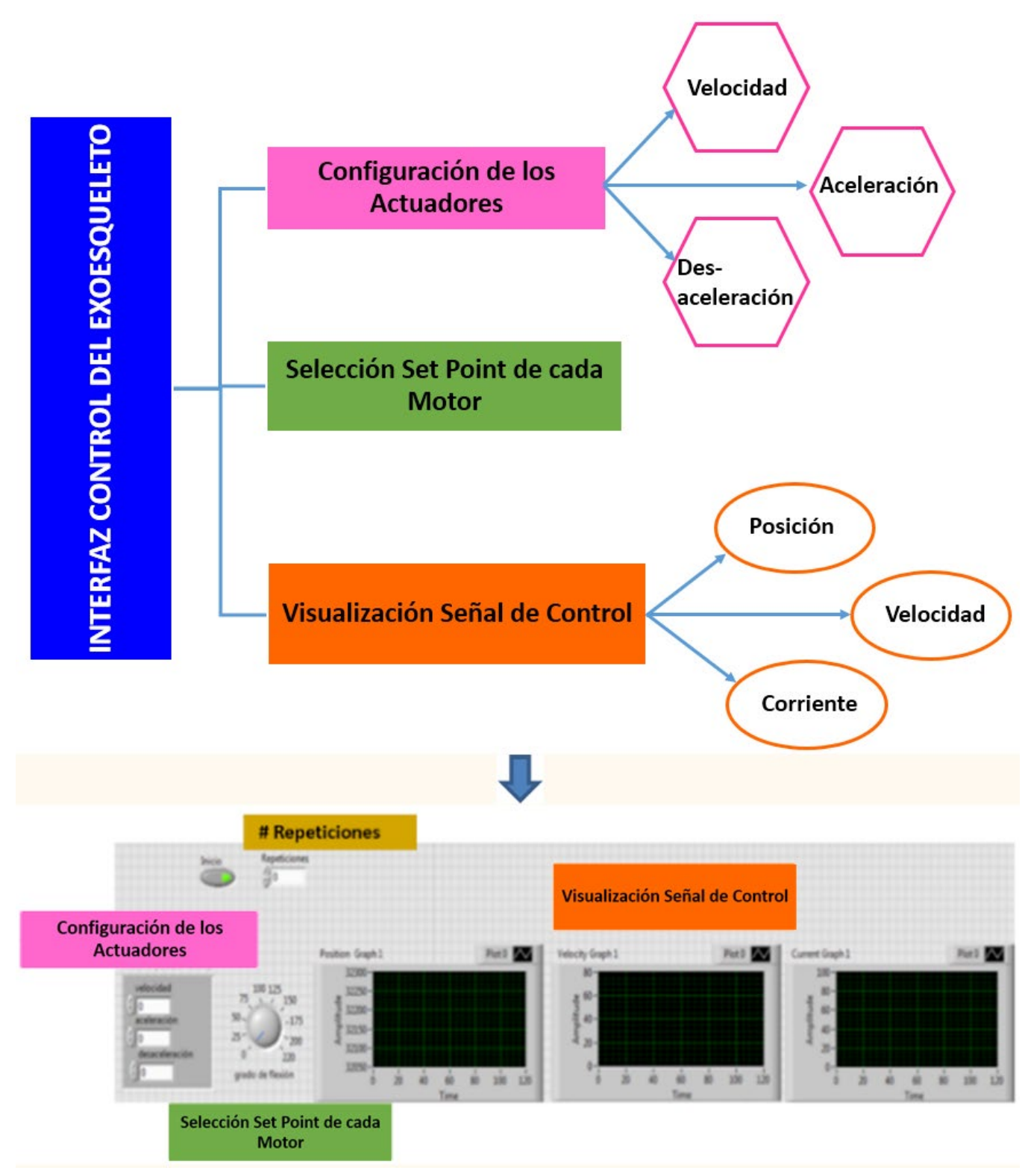

Fig. 11. Interfaz de control de posición.

Fuente: Autores.

Para esto, se ha realizado cinco ciclos de repetición donde fue programada una posición inicial y una posición final teniendo en cuenta los grados de movimiento de la rodilla para este caso. Es importante aclarar que los datos con los que se contaron para realizar este análisis estadístico, se extrajeron del software driver EPOS2, donde la posición de 0 grados corresponde en este caso, al valor de 2.499870 y el valor de -2.300000 corresponde a la posición de -90 grados.

Se realizó una prueba estadística de T para muestras relacionadas con el fin de comparar la posición demandada (programada) respecto a la posición actual (final). En esta prueba estadística se plantean dos hipótesis con la finalidad de conocer cuál es la conclusión del análisis, es así como se definen las hipótesis nula y la alterna:

- Ho = hipótesis nula, donde se considera que no existe diferencias significativas entre la posición demandada y la posición actual.

- $\mathrm{H}_{1}=$ hipótesis alterna, donde se considera que si existe diferencias significativas entre la posición demandada y la posición actual.

Para ello se toma un nivel de significancia de $\mathrm{P}$ valor del $5 \%$ o igual a 0.05 , donde a partir de este estimado se toma la decisión de aceptar o rechazar la hipótesis nula.
La Tabla 6, Tabla 7 y Tabla 8 muestran el resultado de dicho análisis. En este caso, como se observa en la Tabla 6, el promedio de la posición demandada es 2343249,59 en relación con la posición actual que es de 2343259,74 en un mismo periodo de tiempo, lo cual es un incremento bajo y que no tiene una diferencia significativa, para lo cual no afecta al sistema.

Para la comparación entre dos grupos de variables pertenecientes o de muestras relacionadas de un mismo grupo, se considera la prueba de T- students donde la idea es evaluar el antes y el después de una variable, con la finalidad de conocer si el proceso, en este caso, la curva de posición tiene un impacto sobre el movimiento del exoesqueleto durante su trayectoria.

De manera que la prueba seleccionada para este caso de variables que corresponden a T-students se adapta perfectamente; por lo tanto, la intención de esta prueba fue la de conocer si existe una diferencia significativa entre las dos variables del mismo grupo pero en dos ocasiones diferentes.

De acuerdo con resultado obtenido en la significancia bilateral de 0.924 , donde el p-valor está por encima de 0.05 , es pertinente decir que se acepta la hipótesis nula, donde se establece que no existe diferencias significativas entre la posición demandada y la posición actual. 
Tabla 6. Estadísticas de muestras emparejadas.

\begin{tabular}{|c|c|c|c|c|c|}
\hline \multicolumn{2}{|c|}{} & Media & $\mathrm{N}$ & Desviación estándar & Media de error estándar \\
\hline \multirow{2}{*}{ Par 1 } & $\begin{array}{c}\text { Posición Demandada - } \\
\text { Posición Actual }\end{array}$ & $-2343249,59$ & 510 & 68804,221 & 3046,702 \\
\cline { 3 - 6 } & $-2343259,74$ & 510 & 68698,459 & 3042,019 \\
\hline
\end{tabular}

Fuente: Autores.

TABla 7. CoRrelaciones DE MUESTRAS EMPAREJADAS.

\begin{tabular}{|c|c|c|c|c|}
\hline \multicolumn{2}{|c|}{} & N & Correlación & Sig. \\
\hline Par 1 & Posición Demandada - Posición Actual & 510 &, 999 &, 000 \\
\hline
\end{tabular}

Fuente: Autores.

Tabla 8. Prueba de muestras emparejadas.

\begin{tabular}{|c|c|c|c|c|c|c|c|c|c|}
\hline & & & & )iferencias & ejadas & & & & \\
\hline & & Media & Desviación & $\begin{array}{l}\text { Media } \\
\text { de error }\end{array}$ & $\begin{array}{r}95 \% \mathrm{c} \\
\text { confianz }\end{array}$ & $\begin{array}{l}\text { valo de } \\
\text { diferencia }\end{array}$ & $\mathrm{t}$ & gl & $\begin{array}{c}\text { Sig. } \\
\text { (bilateral) }\end{array}$ \\
\hline & & & & estándar & Inferior & Superior & & & \\
\hline Par 1 & $\begin{array}{l}\text { Posición Demandada } \\
\text { - Posición Actual }\end{array}$ & 10,153 & 2406,067 & 106,542 & $-199,164$ & 219,470 & ,095 & 509 & ,924 \\
\hline
\end{tabular}

Fuente: Autores.

\section{CONCLUSIONES}

En el presente artículo se expuso la problemática que algunas personas padecen, luego de sufrir un accidente cerebrovascular y como el desarrollo de algunas tecnologías se puede articular con el cuerpo humano con el propósito de desarrollar sistemas autónomos que sirvan como herramientas para el tratamiento y recuperación en tratamientos de rehabilitación. Aunque al momento del desarrollo de este trabajo no se han hecho pruebas con pacientes humanos, los resultados preliminares con un sistema de pruebas muestran su funcionalidad y potencialidad para el desarrollo de terapias automatizadas.

El sistema desarrollado presenta algunas facilidades que pueden ser útiles en la rehabilitación de pacientes con ictus, entre ellas se tienen las siguientes:

- Se demostró que es posible automatizar el proceso de monitorización y desarrollo de terapias de rehabilitación. Esto trae consigo la ventaja de poder hacer un seguimiento más cercano al desarrollo de los ejercicios por parte de quien diseña la terapia para la recuperación del paciente.

- El sistema es capaz de ayudar en el desarrollo de los ejercicios en los primeros momentos de la recuperación cuando el paciente no tiene la fuerza suficiente para realizar los movimientos. Se espera incluir como mejora la realimentación de fuerza, lo que permitiría hacer que el sistema pueda usarse durante todo el proceso de rehabilitación y permitir al paciente complementar la fuerza requerida para el movimiento hasta su recuperación.
- El diseño mecánico de la estructura, permite el desarrollo de los movimientos naturales de la pierna en el plano sagital y es ajustable a diferentes tipos de pacientes.

- El desarrollo del modelo cinemático y dinámico presentó una buena aproximación entre el sistema simulado y el resultado medido con el prototipo. De la misma manera, este es ajustable de acuerdo a los parámetros biomecánicos del paciente, con lo cual se puede evaluar si el diseño actual con los motores seleccionados permitiría alcanzar los torques para el desarrollo del movimiento en un paciente determinado.

- El sistema presenta una interfaz básica desarrollada en Labview, en la cual se pueden especificar los parámetros básicos para el desarrollo de la terapia y su registro. Esta interfaz sigue actualmente en desarrollo y se esperan incluir mejoras que permitan al usuario o al fisioterapeuta tener mayor información del proceso de recuperación.

\section{REFERENCIAS}

[1] "Disability," Banco Mundial, NW Washington, DC USA, Abril 3, 2019. [Online]. Available: https://www.bancomundial.org/es/topic/disability

[2] H. Collado, Situación Mundial de la discapacidad, Tegucigalpa, HN: Universidad Nacional Autónoma de Honduras, OPS, 2013. [Online]. Available: http://www.bvs.hn/Honduras/Discapacidad/Docentes/Situacion\%20Mundial\%20 de\%20la\%20Discapacidad.pdf

[3] J. A. Miranda, "Análisis de las dificultades, desafíos y propuestas de mejora en el proceso de inclusión laboral efectivo en personal con discapacidad auditiva," Tesis de Grado, Dept. de Ing. Ind., Univ. Técn. Federico Santa María, Valparaíso. Chile, 2017. 
[4] J. Gómez, Identificación de las personas con discapacidad en los territorios desde el rediseño del registro. Bogotá, Colombia: Departamento Administrativo Nacional de Estadística, 2008. [Online]. Available: http://www.dane.gov. co/files/investigaciones/discapacidad/identificacion $\% 20$ en\%20los\%20territorios.pdf

[5] F. Silva, J. Zarruk, C. Quintero, W. Arenas, F. Cristian, S. Rueda \& A. Estupiñán, "Enfermedad cerebrovascular en Colombia", Rev. Col. Cardiol., vol. 13, no. 2, pp. 85-89, Sept-Oct. 2006.

[6] República de Colombia. Ministerio de Salud y Protección Social, Oficina de Promoción Social. (2018, Septiembre). Sala situacional de las Personas con Discapacidad. [Online]. Available: http://ondiscapacidad.minsalud.gov.co/Documentos\%20compartidos/Sala\%20situación\%20Discapacidad\%20septiembre\%202018.pdf

[7] República de Colombia. Consejo Nacional de Política Económica y Social. Departamento Nacional de Planeación. (2013, Diciembre 9). Política pública nacional de discapacidad e inclusión social. Conpes Social 216. [Online]. Available: https://www.minsalud.gov.co/sites/rid/Lists/BibliotecaDigital/RIDE/INEC/IGUB/CONPES166.pdf

[8] C. Velandia, H. Celedón, D. A. Tibaduiza, C. Torres-Pinzón \& J. Vitola, "Design and control of an exoskeleton in rehabilitation tasks for lower limb," presented at the XXISymposium on Signal Processing, Images and Artificial Vision (STSIVA), Bucaramanga, Colombia, Aug. 31-Sept. 2, 2016, pp. 1-6. http://dx.doi.org/10.1109/STSIVA.2016.7743341

[9] P. Aya, "Estudio anatómico y determinación de parámetros funcionales de un prototipo de exoesqueleto de miembro inferior con dos grados de libertad", Master thesis, Fac. Ing. Electrónica. Univ. Santo Tomás, Bogotá, Colombia, 2017.

[10] Y. Lee, Y. J. Kim, J. Lee, M. Lee, B. Choi, J. Kim, Y. J. Park, \& J. Choi, "Biomechanical Design of a Novel Flexible Exoskeleton for Lower Extremities," IEEE/ASME Transactions on Mechatronics, vol. 22, no. 5, pp. 2058-2069, Oct. 2017. http://dx.doi.org/10.1109/TMECH.2017.2718999

[11] Z. F. Lerner, D. L. Damiano, H. S. Park, A. J. Gravunder, \& T. C. Bulea, "A Robotic Exoskeleton for Treatment of Crouch Gait in Children With Cerebral Palsy: Design and Initial Application," IEEE Transactions on Neural Systems and Rehabilitation Engineering, vol. 25, no. 6, pp. 650-659, Jun. 2017. http://doi.org/10.1109/TNSRE.2016.2595501

[12] S. Kim, \& J. Bae, "Force-Mode Control of Rotary Series Elastic Actuators in a Lower Extremity Exoskeleton Using Model-Inverse Time Delay Control," IEEE/ASME Transactions on Mechatronics, vol. 22, no. 3, pp. 1392-1400, Jun. 2017. http://doi.org/10.1109/TMECH.2017.2687979

[13] A. M. Dollar, \& H. Herr, "Lower Extremity Exoskeletons and Active Orthoses: Challenges and State of the Art", IEEE Transactions on Robotics, vol. 24, no. 1, pp. 144158. Feb. 2008. http://doi.org/10.1109/TRO.2008.915453

[14] M. Vukobratović, "Active exoskeletal systems and beginning of the development of humanoid robotics," Facta universitatis-series: Mechanics, Automatic Control and Robotics, vol. 7, no. Special, pp. 243-262. Mar. 2008.

[15] W. Huo, S. Mohammed, J. C. Moreno, \& Y. Amirat, "Lower limb wearable robots for assistance and rehabilitation: A state of the art," IEEE Systems Journal, vol. 10, no. 3, pp. 1068-1081. Oct. 2014. http://dx.doi.org/10.1109/ JSYST.2014.2351491

[16] A. Zoss, \& H. Kazerooni, "Design of an electrically actuated lower extremity exoskeleton," Advanced Robotics, vol. 20, no. 9. pp. 967-988. Mar. 2006. https://doi. org/10.1163/156855306778394030
[17] J. Pratt, P. Dilworth, \& G. Pratt, "Virtual model control of a bipedal walking robot," presented at the Proc. IEEE ICRA, Robotics and Automation, Albuquerque, NM, USA, 20-25 Apr. 1997, pp. 193-198. http://dx.doi. org/10.1109/ROBOT.1997.620037

[18] J. F. Veneman, R. Kruidhof, E. E. G. Hekman, R. Ekkelenkamp, E. H. F. Van Asseldonk, \& H. van der Kooij, "Design and evaluation of the lopes exoskeleton robot for interactive gait rehabilitation," IEEE Trans. Neural Systems and Rehabilitation Engineering, vol. 15, no. 3. pp. 379-386, Oct. 2007.

[19] C. Velandia, H. Celedón, D. Tibaduiza, C. Torres, \& J. Vitola, "Design and control of an exoskeleton in rehabilitation tasks for lower limb," presented at the XXI Symposium on signal processing, images and artificial visión (STSIVA), Bucaramanga, Colombia, Aug. 31 - Sept. 2, 2016. http://dx.doi.org/10.1109/STSIVA.2016.7743341

[20] C. Fleischer, \& G. Hommel. "Torque control of an exoeskeletan knee whith EMG signals," presented at the Proc. Joint conference on robotics, 37th International Symposium on Robotics and Robotik 2006, Munich, Germany, May. 15-17, 2006.

[21] J. M Font Llagunes, G Arroyo, F. J. Alonso, \& B. M. Vinagre, "Diseño de una órtesis activa para ayuda a la marcha de lesionados medulares", presentado en XVIII Congr. Nac. de Ing. Mec., CNIM 2010, Ciudad Real, España, Nov. 3-5, 2010.

[22] M. Barnes, "The Advancement of Human Motion Control," Product Desing \& Development, vol. 68, no 5, pp. 32-33, Jun. 2013.

[23] W. Johnson, O. Onuma, M. Owolabi, \& S. Sachdev, "Stroke: a global response is needed," Bull World Health Organ, vol. 94, no. 9, pp. 634-634A, Sept. 2016. http:// dx.doi.org/10.2471/BLT.16.181636

[24] C. Melchiorri, "Dynamic Model of Robot Manipulators," Ind. Robot., Dipt. di ing., energia elett. e Dell'informazione. Universita di bologna, Italy.

[25] P. Sánchez, \& M. A. Arteaga-Pérez, "Simplied Methodology for Obtaining the Dynamic Model of Robot Manipulators," International Journal of Advanced Robotic Systems, vol. 9, no. 5, pp. 1-12, Jan, 2012. http://dx.doi. org/10.5772/51305

[26] EPOS2 50/5, Digital positioning controller, 5 A, 11-50 VDC. Maxon Group, Sachseln, Suiza. [Online]. Available: http://www.maxonmotor.com

[27] MILE Encoder for EC 45 flat. Maxon Group, Sachseln, Suiza. [Online]. Available: http://www.maxonmotor.co.uk

[28] C. Velandia, D. A. Tibaduiza, \& M. Anaya, "Proposal of Novel Model for a 2 DOF Exoskeleton for Lower-Limb Rehabilitation," Robotics, vol. 6, no. 3, pp. 1-25, Aug. 2017. http://dx.doi.org/10.3390/robotics6030020

[29] J. M. Grosso, "Diseño y validación de un exoesqueleto de piernas de tipo maestro-esclavo para facilitar la rehabilitación de personas con discapacidad parcial de su locomoción en el plano sagital", Tesis de pregrado, Fac. Ing. Mecatrónica. Univ. Autónoma de Bucaramanga, Santander, Colombia, 2008.

[30] J. M. Grosso, \& D. A. Tibaduiza. "Diseño conceptual de un exoesqueleto para asistir la rehabilitación de miembro inferior," presentado en III Congr. Int. Ing. Mecatrónica UNAB, CIIMECA 3, Bucaramanga, Colombia, Oct. 5-7, 2011

[31] D. A. Tibaduiza, N. Chio, J. M. Grosso, \& M. Anaya, "Diseño conceptual de un exoesqueleto para asistir la rehabilitación de miembro inferior," en III Congr. Int. Ing. Mecatrónica UNAB, CIIMECA 3, Bucaramanga, Colombia, Oct. 5-7, 2011. 
[32] J. M. Grosso, \& D. A. Tibaduiza, "Simulación cinemática de un exoesqueleto de brazo", presentado en II Congr. Int. Ing. Mecatrónica, CIIMECA 2, Bucaramanga, Colombia, Sept. 30-Oct. 2, 2009.

[33] D. A. Tibaduiza, N. Chio, J. M. Grosso, \& M. Anaya, "Diseño de un exoesqueleto mecatrónico de brazo basado en screws y robots paralelos," presentado en II Congr. Int. Ing. Mecatrónica, CIIMECA 2, Bucaramanga, Colombia, Sept. 30-Oct. 2, 2009.

[34] J. M. Grosso, \& D. A. Tibaduiza, "Diseño y validación de un exoesqueleto maestro-esclavo para rehabilitación de piernas," presentado en VI Congr. Int. de Investigaciones en Ing. Eléctrica y Electrónica, CIIIEE 2008, Aguascalientes, México, Nov. 3-7, 2008.

[35] C. Velandia. "Modelado, control y monitoreo de un exoesqueleto para asistir procesos de rehabilitación en miembro inferior," Tesis de pregrado, Fac. Ing. Elect., Univ. Santo Tomás, Bogotá, Colombia, 2017. http://dx.doi.org/10.13140/ RG.2.1.1280.9205

[36] J. F. Ayala, G. Urriolagoitia, B. Romero, C. R. Torres, L. A. Aguilar, \& G. M. Urriolagoitia-Calderón, "Diseño mecánico de un exoesqueleto para rehabilitación de miembro superior," Revista Colombiana de Biotecnología, vol. 17, no. 1, pp. 79-90, Ene. 2015. http://doi.org/10.15446/rev.colomb.biote.v17n1.44188

[37] R. López, H. Aguilar, S. Salazar, R. Lozano \& J. Torres, "Modelado y control de un exoesqueleto para rehabilitación de extremidad inferior con dos grados de libertad," Revista Iberoamericana de Automática e Informática Industrial, vol. 11, no. 3, pp. 304-314, Jul.-Sept., 2014. https://doi.org/10.1016/j.riai.2014.02.008

[38] H. Celedón, "Diseño mecatrónico de un robot exoesqueleto de extremidad superior para rehabilitación de personas con discapacidad parcial en el codo," Tesis de pregrado, Fac. Ing. Elect. Univ. Santo Tomás, Bogotá, Colombia, 2016.

[39] R. Gutierrez, F. Vanegas, J. Duque, O. Avilés \& P. Niño, "Diseño y control de un exoesqueleto para rehabilitación motora en miembro superior," in Proc. IV Latin American Congress on Biomedical Engineering 2007, Bioengineering Solutions for Latin America Health, C. MüllerKarger, S. Wong \& A. Cruz, Eds., Margarita Island, Ven, Springer, 2007, pp. 758-761.

[40] J. Loja \& A. Ordoñez, "Diseño y construcción de un exoesqueleto de rodilla robótica para asistir a pacientes en etapas de rehabilitación", Tesis de pregrado, Fac. de Ing., Univ. de Cuenca, Azuay, Ecuador, 2016.

[41] X. Zhang, Z. Yue, \& J. Wang, "Robotics in-Lowerlimb rehabilitation after stroke," Behavioural Neurology, vol. 2017, no. 4, pp. 1-13, Jun. 2017. https://doi. org/10.1155/2017/3731802

[42] T. Zhang, M. Tran, \& H. Huang, "Design and experimental verification of hip exoskeleton with balance capacities for walking assistance," IEEE/ASME Transaction on Mechatronics, vol. 23, no. 1, pp. 274-285. Feb. 2018. http:// dx.doi.org/10.1109/TMECH.2018.2790358

[43] T. M. Schnieders, \& R. T. Stone, "A current review of human factors and ergonomic intervention with exoskeletons," in Novel Design and applications of robotics technologies, D. Zhang, \& B. Wei, Eds., Hershey, PA: IGI Global, 2019, pp. 217-246. http://dx.doi.org/10.4018/978-1-52255276-5.ch008
Diego Alexander Tibaduiza-Burgos es Ingeniero y Magíster en Ingeniería Electrónica de la Universidad Industrial de Santander (Colombia) con Doctorado en Ingeniería Sísmica y Dinámica Estructural de la Universitat Politécnica de Catalunya (España). https:// orcid.org/0000-0002-4498-596X

Pedro Antonio Aya Parra es Ingeniero Biomédico y Magister en Ingeniería Electrónica con énfasis en Robótica y Automatización de la Universidad Santo Tomas (Colombia). Su interés en investigación está centrado en el área de Rehabilitación, Ingeniería Clínica y Bioinstrumentación. Investigador de la facultad de Ingeniería Biomédica de la Escuela de Medicina y Ciencias de la Salud de la Universidad del Rosario (Colombia) y asociado al grupo de investigación Gibiome (Colciencias). https://orcid.org/0000-0002-3987-1028

Maribel Anaya Vejar es Ingeniero y Magíster en Ingeniería Electrónica de la Universidad Industrial de Santander (Colombia) con doctorado en Ingeniería Sísmica y Dinámica Estructural de la Universitat Politecnica de Catalunya (España). https://orcid. org/0000-0002-0241-4771 